\title{
A multicenter single-arm trial of sintilimab in combination with chemotherapy for neoadjuvant treatment of resectable esophageal cancer (SIN-ICE study)
}

\author{
Hongtao Duan ${ }^{1 \#}$, Tianhu Wang ${ }^{2 \#}$, Zhilin Luo ${ }^{2 \#}$, Xiaoyuan Wang ${ }^{3 \#}$, Honggang Liu ${ }^{1}$, Liping Tong ${ }^{1}$, \\ Xiaoping Dong ${ }^{1}$, Yong Zhang ${ }^{1}$, Michele Valmasoni ${ }^{4}$, Biniam Kidane ${ }^{5}$, Khaldoun Almhanna ${ }^{6}$, Ory Wiesel ${ }^{7}$, \\ Sainan Pang ${ }^{3}$, Jianqun $\mathrm{Ma}^{3}$, Xiaolong Yan ${ }^{1}$ \\ ${ }^{1}$ Department of Thoracic Surgery, Tangdu Hospital, Air Force Medical University, Xi'an, China; ${ }^{2}$ Department of Thoracic Surgery, Third Affiliated \\ Hospital, Chongqing Medical University, Chongqing, China; ${ }^{3}$ Thoracic Surgery Department, Harbin Medical University Cancer Hospital, Harbin, \\ China; ${ }^{4}$ Department of Surgery, Oncology and Gastroenterology, School of Medicine, University of Padova, Padova, Italy; ${ }^{5}$ Section of Thoracic \\ Surgery, University of Manitoba, Winnipeg, Manitoba, Canada; ${ }^{6}$ Division of Hematology/Oncology, The Warren Alpert Medical School of Brown \\ University, Lifespan Cancer Institute, Rhode Island Hospital, Providence, RI, USA; ${ }^{7}$ Division of Foregut and Thoracic Surgery, Department of \\ Surgery, Rabin Medical Center, Beilinson Hospital, Petah Tikva, Israel \\ Contributions: (I) Conception and design: J Ma, X Yan, S Pang, T Wang; (II) Administrative support: J Ma, X Yan, H Liu, T Wang; (III) Provision \\ of study materials or patients: H Duan, Z Luo, X Wang; (IV) Collection and assembly of data: L Tong, X Dong, Y Zhang; (V) Data analysis and \\ interpretation: L Tong, X Dong, Y Zhang; (VI) Manuscript writing: All authors; (VII) Final approval of manuscript: All authors. \\ "These authors contributed equally to this work. \\ Correspondence to: Sainan Pang; Jianqun Ma. Thoracic Surgery Department, Harbin Medical University Cancer Hospital, 150 Haping Road, Nangang \\ District, Harbin 150081, China. Email: psn@hrbmu.edu.cn; jianqunma@aliyun.com. Xiaolong Yan. Department of Thoracic Surgery, Tangdu \\ Hospital, Air Force Medical University, 1 Xinsi Road, Qiaoba, Xi’an 710038, China. Email: yanxiaolong@fmmu.edu.cn.
}

Background: Preoperative chemotherapy or chemoradiotherapy is the standard treatment for resectable esophageal cancer (EC); however, it is associated with increased postoperative complications and mortality. Recently, Immune Checkpoint inhibitors have been incorporated in the treatment of advanced EC. Its role in the preoperative setting has not been established yet. In this multicenter, single-arm study, we evaluated the efficacy and safety of neoadjuvant therapy with sintilimab in combination with chemotherapy in treating EC.

Methods: Patients received neoadjuvant therapy with 3 cycles of sintilimab $200 \mathrm{mg}$ Q3W in combination with platinum-based chemotherapy. Surgery was performed within 4-6 weeks after neoadjuvant therapy. The primary endpoints of the trial were pathological complete response (pCR) and safety.

Results: A total of 23 patients (21 men and 2 women) were enrolled. Surgery was completed in 17 participants, with 16 achieving R0 resection and 1 had R1 resection, 5 participants refused surgery. One patient progressed prior to surgery. Twenty one patients (91\%) had significant improvement in their dysphagia following treatment as assessed by Stooler's criteria. The majority of patients who underwent resection have a good pathological response and downstaging rate was $76.5 \%$ (13/17). A pCR was achieved in 6 cases $(6 / 17,35.3 \%)$ and major pathological response (MPR) in 9 cases $(9 / 17,52.9 \%)$. The main preoperative adverse events (AEs) were vomiting (13/23, 56.5\%), leukopenia (12/23, 52.2\%), neutropenia $(9 / 23,39.1 \%)$, and malaise $(8 / 23,34.8 \%)$. Immune-related AEs were mild and included hypothyroidism $(2 / 23,8.7 \%)$ and rash $(4 / 23,17.4 \%)$. The incidence of $\geq$ grade 3 treatment related AEs was $30.4 \%(7 / 23)$. There were no $\geq$ grade 4 AEs.

Conclusions: Sintilimab in combination with chemotherapy in the neoadjuvant treatment of EC is safe and lead to a high pCR. Therefore, further testing is warranted.

Keywords: Sintilimab; pathological complete response (pCR); major pathological response (MPR); safety; efficacy 
Submitted Aug 26, 2021. Accepted for publication Nov 24, 2021.

doi: $10.21037 / \mathrm{atm}-21-6102$

View this article at: https://dx.doi.org/10.21037/atm-21-6102

\section{Introduction}

Esophageal cancer (EC) is the sixth most common cause of cancer-related death worldwide and the sixth most common cancer in China (1). Despite recent improvements in treatment, the prognosis of EC is still poor. Immune checkpoint inhibitors have changed the landscape of cancer treatment in several malignancies including EC. Although not considered valid predictive markers in other malignancies, it has been reported that up to $44 \%$ of EC patients express PD-1 with a combined positive score (CPS) $>1 \%$ (1).

Incorporating immune checkpoint inhibitors in the treatment of EC has been the subject off several clinical trials. In KEYNOTE-181 study, where patients with advanced EC who progressed on first line treatment were randomized to either second line chemotherapy $v$ s. pembrolizumab; treatment with single agent pembrolizumab was superior to chemotherapy with 12 -month overall survival (OS) rate of $43 \%$, compared to $20 \%$ in the chemotherapy arm (2). More recently, KEYNOTE-590 study randomized treatment naive patients with advanced EC to chemotherapy plus minus pembrolizumab; the objective response rate (ORR) was of $45 \%$ and a median progression-free survival (PFS) of 8.3 months in the chemotherapy plus pembrolizumab arm versus 9.3 and 6.3 months in the chemotherapy only arm respectively. Patients with esophageal squamous cell carcinoma (ESCC) have a median OS of 12.6 months in the combination arm which was nearly 2.8 months longer than that in the chemotherapy plus placebo group. ESCC patients with a high CPS (>10) had an even longer OS (13.9 months in the combination arm) which was 5.1 months longer than that in the chemotherapy plus placebo group (3).

Sintilimab, a recombinant humanized anti-PD-1 monoclonal antibody, has been approved in China for the treatment of Melanoma and Hepatocellular carcinoma among others and is currently being developed for use in various malignancies, including non-small cell lung cancer and EC. Several ongoing trials are underway in the United States as well. In a recent open label phase I study, the combination of sintilimab plus chemotherapy in patient with advanced lung cancer was found to be safe and showed an ORR of $68.4 \%$ and $64.7 \%$ in adenocarcinoma and squamous carcinoma, respectively (4). In a another study, patients with resectable non-small cell lung cancer (NSCLC) (stage IAIIIB) received two cycles of sintilimab prior to surgery, out of 40 treated patients, six patients (16\%) achieved pathological complete response (pCR) and 15 (40.5\%) achieved major pathological response (MPR) (5). Treatment with sintilimab was well tolerated and considered safe and feasible in the neoadjuvant treatment. Several recently reported studies have shown safety and efficacy of adding PD-1 antibodies to chemotherapy in the neoadjuvant setting. In the phase II NADIM trial (neoadjuvant chemotherapy and nivolumab in resectable non-small-cell lung cancer), the combination of nivolumab plus chemotherapy in patients with resectable NSCLC lead to a pCR of $63 \%$ (6). In our previous study, the pCR after neoadjuvant treatment with PD-1 inhibitor combined with chemotherapy in lung cancer patients reached $33.7 \%$ (7). All above trials supported that combining PD-1 inhibitors with chemotherapy is safe and feasible in the neoadjuvant setting.

Neoadjuvant chemoradiotherapy has been established as the standard of care in patients with resectable EC based on the CROSS trial (8). However, concurrent chemoradiotherapy can be toxic and might increase postoperative morbidity and mortality (9). Several ongoing trials (i.e., Neo-AEGIS "Neoadjuvant trial in Adenocarcinoma of the Esophagus and Esophago-Gastric Junction International Study") are challenging the role of radiation in the neoadjuvant setting and final results are currently awaited (10). The addition of PD-1 inhibitors to neoadjuvant chemoradiation is currently being evaluated as well. In the recently reported PALACE-1 (preoperative pembrolizumab combined with chemoradiotherapy for resectable esophageal squamous cell carcinoma) trial, treatment was found to be safe with a pCR of 55.6\% (10/18), but grade III and higher AEs were observed (13/20, 65\%) and the most frequent grade III AEs was lymphopenia (12/13, $92 \%)(11)$. Similar results were seen in a multicenter study adding pembrolizumab to neoadjuvant chemoradiation in patients with esophageal adenocarcinoma followed by a year of pembrolizumab (12). Based on the above, we conducted a multicenter, single-arm, open-label trial to evaluate the safety and efficacy of adding sintilimab to standard chemotherapy in the neoadjuvant therapy of resectable EC, aiming to identify a safer treatment modality. We present the following article 
in accordance with the TREND reporting checklist (available at https://dx.doi.org/10.21037/atm-21-6102).

\section{Methods}

\section{Study design (single-arm)}

\section{Inclusion criteria}

The trial was conducted in three centers in China: The Department of Thoracic Surgery of the Third Affiliated Hospital of Chongqing Medical University, the Thoracic Surgery Department, Harbin Medical University Cancer Hospital, and the Department of Thoracic Surgery of Tangdu Hospital of Air Force Medical University. All procedures performed in this study involving human participants were in accordance with the Declaration of Helsinki (as revised in 2013). The treatment protocol was approved by the Ethics Committee of Tangdu Hospital of the Air Force Medical University (approval number: 202005-12-KY-07-XW-01), and all participants and their families signed informed consent forms. The inclusion criteria were as follows: (I) age $\geq 18$ years; (II) pathologically confirmed ESCC; (III) resectable clinical $\mathrm{T}_{2-\mathrm{x}} \mathrm{N}_{\mathrm{x}} \mathrm{M}_{0}$, as assessed by chest contrast-enhanced computed tomography (CT) or positron emission tomography-computed tomography (PET-CT); (IV) adequate pre-operative cardiac and lung functions, as demonstrated by lung function tests, blood gas analysis, and cardiac color ultrasound. The exclusion criteria included active autoimmune disease, active concurrent malignancy, ongoing systemic steroids use (>10 mg daily prednisone equivalents), and known acquired immune deficiency syndrome (AIDS).

\section{Treatment protocol}

The participants received neoadjuvant therapy with 3 cycles of sintilimab $200 \mathrm{mg}$ Q3W in combination with platinum-based chemotherapy (docetaxel $75 \mathrm{mg} / \mathrm{m}^{2}$ on day 1 , albumin-bound paclitaxel $130 \mathrm{mg} / \mathrm{m}^{2}$ on days 1 and 8 or $260 \mathrm{mg} / \mathrm{m}^{2}$ on day 1 , plus nedaplatin $80 \mathrm{mg} / \mathrm{m}^{2}$ on day 1). Surgery was performed within 4-6 weeks after the neoadjuvant therapy. McKeown esophagectomy or IvorLewis esophagectomy was performed, with lymph node (LN) dissection in at least two fields.

\section{Primary endpoints}

The primary endpoints of the trial were safety and pCR. Preoperative adverse events (AEs) were assessed using the Common Terminology Criteria Adverse Events (CTCAE) version 4.0 (https://evs.nci.nih.gov/ftp1/CTCAE/ CTCAE_4.03/Archive/CTCAE_4.0_2009-05-29_ QuickReference_8.5x11.pdf), and postoperative complications.

\section{Secondary endpoints}

\section{Efficacy}

(I) According to the Stooler criteria, the symptoms of dysphagia were graded on a scale of 0-4: $0=$ normal solid food; $1=$ dysphagia to soft food; $2=$ dysphagia to semiliquids; $3=$ dysphagia to liquids; and $4=$ inability to swallow saliva. Any symptom improvement by 1 grade over the previous one was considered as symptom remission. (II) The ORR was calculated according to the RECIST guidelines version 1.1 (https://recist.eortc.org/recist-1-1-2/). Complete response (CR) was declared when all the lesions (including scars) had disappeared radiologically or under gastroscope, partial response $(\mathrm{PR})$ when there was a decrease in the size of target lesion (short diameter of $\mathrm{LN}>15 \mathrm{~mm}$ ) $\geq 30 \%$, stable disease (SD) when change in target lesions was within $\pm 20 \%$, progressive disease (PD) when the lesion enlarged by $20 \%$; a judgment of "Not Evaluated" was made if there was no target lesion and the primary lesion did not reach PR, recorded as NON-CR/NON-PD. (III) Pretreatment clinical staging was determined using contrastenhanced CT; LNs with a short diameter greater than $1 \mathrm{~cm}$ were considered positive. (IV) Pathological response was evaluated following surgery. pCR was defined as the absence of residual invasive cancer (ypTONOM0). A MPR was defined as a residual viable tumor (RVT) of less than or equal to $10 \%$ of the specimen. Pathological downstaging was defined as a decrease in the extent of tumor presence (ypTNM) after treatment compared with baseline.

\section{PD-1 expression}

Programmed death ligand 1 (PD-L1) expression was assessed as CPS by immunohistochemistry in tumor samples obtained at initial diagnosis (SP263).

\section{Statistical analysis}

Continuous variables were presented as mean \pm standard deviation, and categorical data were presented as percentages. Variables were compared using $t$-test, chi-square test/ analysis of variance (ANOVA), or Fisher's exact test. The potential correlation of PD-L1 expression with RVT was 
Page 4 of 8

Duan et al. Sintilimab/chemotherapy as neoadjuvant therapy in ESCC

Table 1 Clinical features

\begin{tabular}{|c|c|}
\hline Variable & Value \\
\hline Age (years) & $63.5[56-81]$ \\
\hline \multicolumn{2}{|l|}{ Gender, n (\%) } \\
\hline Male & $21(91.3)$ \\
\hline Female & $2(8.7)$ \\
\hline \multicolumn{2}{|l|}{ ECOG, n (\%) } \\
\hline 0 & $21(91.3)$ \\
\hline 1 & $2(8.7)$ \\
\hline \multicolumn{2}{|c|}{ Tumor location, n (\%) } \\
\hline Proximal third & $1(4.3)$ \\
\hline Middle third & 19 (82.6) \\
\hline Distal third & $3(13.0)$ \\
\hline \multicolumn{2}{|c|}{ Clinical stage, n (\%) } \\
\hline II & $4(17.4)$ \\
\hline III & $17(73.9)$ \\
\hline IVA & $2(8.7)$ \\
\hline \multicolumn{2}{|l|}{ Clinical T stage } \\
\hline 2 & $1(4.3)$ \\
\hline 3 & $20(87.0)$ \\
\hline 4 & $2(8.7)$ \\
\hline \multicolumn{2}{|c|}{ Clinical N stage, n (\%) } \\
\hline 0 & $5(21.7)$ \\
\hline 1 & $12(52.2)$ \\
\hline 2 & $6(26.1)$ \\
\hline
\end{tabular}

ECOG, Eastern Cooperative Oncology Group.

analyzed by using Pearson' $t$-test in the SPSS 19.0 software package (IBM Corp., Armonk, NY, USA). A P value less than 0.05 was considered to be statistically significant. We made the assumption that treatment would not be feasible if the probability that the minimum acceptable pCR was $20 \%$ compared to the prospective study. The study is the exploratory nature, so 23 patients was determined.

\section{Results}

\section{Efficacy}

A total of 23 patients (21 men and 2 women) were enrolled. There are 4, 17 and 2 patients with clinical stage II, III and IV respectively. According to the Stooler criteria, the dysphagia grade was 2 in 17 cases and 3 in 5 cases at diagnosis. The mean age was 63.5 years (range, $56-81$ years) (Table 1). Seventeen patients underwent surgical resection with 16 achieving R0 resection. No R2 resection was noted. Two patients received only one cycle of treatment and achieved MPR after surgery. A total of 5 participants refused surgery after resolution of their symptoms; they went to receive altogether 4-6 cycles of chemotherapy plus sintilimab followed by sintilimab monotherapy for maintenance therapy until disease progression. One participant dropped out due to disease progression. As of the last follow-up date (15 June 2021) the mean disease-free survival (DFS) was 13.8 months (range, 7.1-24.2 months) for the surgical participants, and 1 surgical participant died due to tumor progression; the DFS was 10.1 months (range, 4.0-12.1 months) for the non-surgical patients (Figure 1). According to the RECIST 1.1 imaging appendix, 8 participants were evaluable for clinical response, among whom there were 3 cases of CR, 2 cases of PR, 2 cases of $\mathrm{SD}$, and 1 case of $\mathrm{PD} ; 15$ participants were non-evaluable (NON-CR/NON-PD). The ORR was $66.7 \%$ (for evaluable participants only). Following treatment, dysphagia was markedly alleviated in the 21 participants (91.3\%), the dysphagia grade improved significantly based on the Stooler criteria and was 3 in 1 case, 2 in 1 case and 0 in 17 cases. After the surgery, it's been showed pathological stage I included 10 patients, stage II for 4 patients, stage IIIA for 1 patient, and stage IIIB for 2 patients. So, pathological downstaging was $76.5 \%(13 / 17)$. In the 17 patients who underwent surgery, compete pathological response (pCR) was achieved in 6 patients $(35.3 \%$ ) and MPR in $9(52.9 \%)$. McKeown esophagectomy was performed in 15 participants, Ivor-Lewis esophagectomy in 1 case, and Sweet esophagectomy in 1 patient who could not tolerate the above procedures due to poor nutritional status. The mean operative time was $350.9 \mathrm{~min}$ (range, $185-550 \mathrm{~min}$ ). The estimated blood loss was $165 \mathrm{~mL}$ (range, $50-500 \mathrm{~mL}$ ). The average number of LNs removed was 25.3 [10-43]. The average length of hospital stay was 18.5 days (range, 8-92 days) (Table 2).

The correlation between PD-L1 expression and pathological response was evaluated. Correlation analysis showed no correlation between PD-L1 expression and RVT after neoadjuvant therapy $(\mathrm{r}=0.06, \mathrm{P}=0.86)$. In addition, there was no significant difference in pCR between PD-L1 positive group $(\mathrm{CPS} \geq 1)$ and $\mathrm{PD}-\mathrm{L} 1$ negative group (CPS $<1)(\mathrm{P}=0.196)$ (Figure 2). 


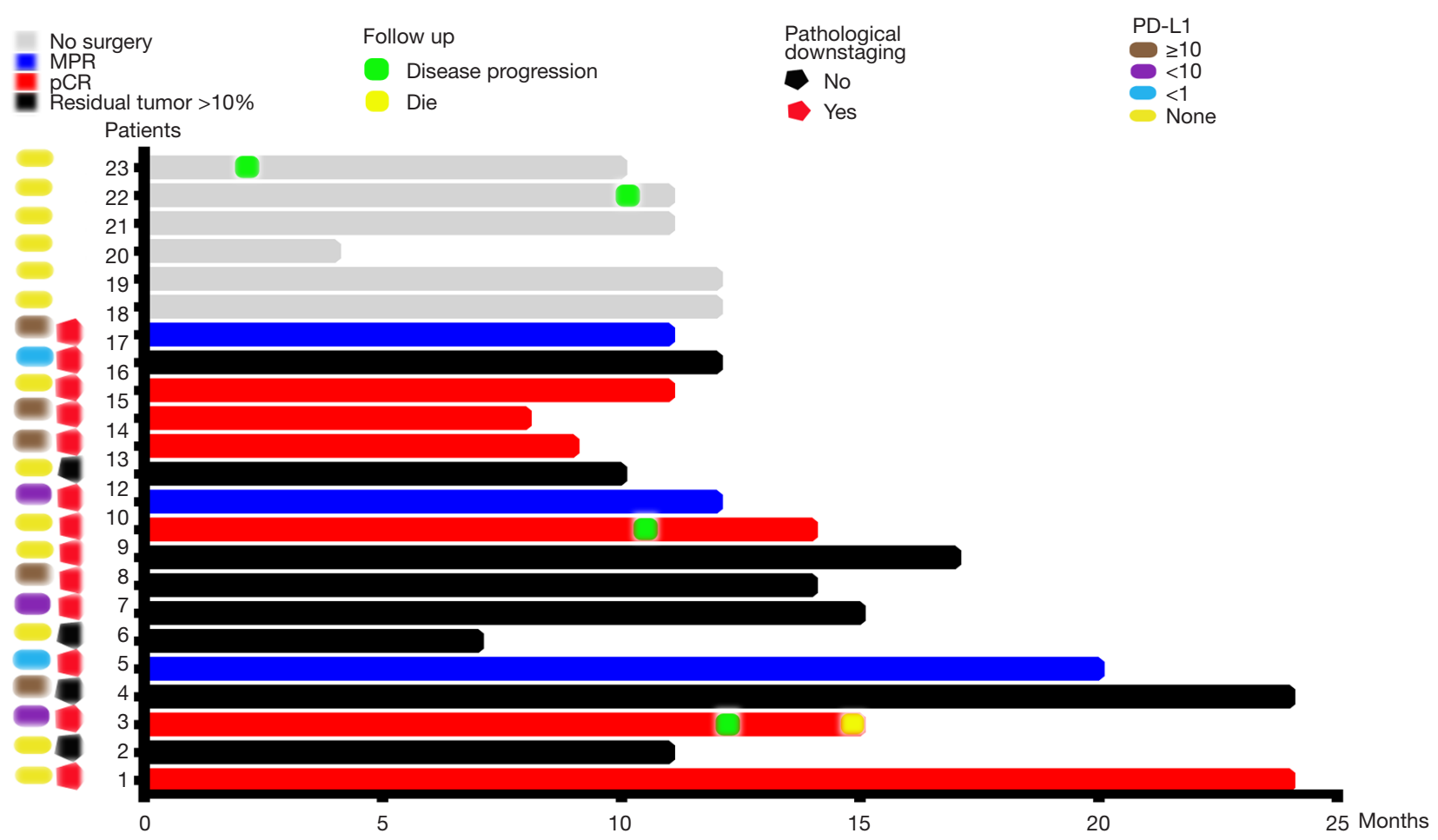

Figure 1 Swimlane diagram PFS in the intention-to-treat population ( $\mathrm{n}=23)$. Each lane represents one patient. The left column shows some of the clinical features. PFS, progression-free survival; pCR, pathological complete response; MPR, major pathological response; PD-L1, programmed death-ligand 1 .

\section{Safety}

Safety assessment was based on preoperative AEs (according to CTCAE 4.0) and postoperative complications. The main preoperative AEs were vomiting (13/23, 56.5\%), leukopenia $(12 / 23,52.2 \%)$, neutropenia (9/23, 39.1\%), and malaise $(8 / 23,34.8 \%)$. Immune-related AEs included hypothyroidism $(2 / 23,8.7 \%)$ and rash $(4 / 23,17.4 \%)$. The incidence of $\geq$ grade 3 AEs was $30.4 \%$ (7/23). No $\geq$ grade $4 \mathrm{AE}$ or death was noted. There were no surgical delays (Table 3). There were no 90-day perioperative deaths; 2 participants experienced anastomotic leaks, which were treated and resolved. Other relatively common complications were pneumonia $(n=6)$, hoarseness $(n=5)$, heart failure $(n=4)$, and respiratory failure $(n=2)$ (Table 2). There was no clear correlation between neoadjuvant treatment and complications.

\section{Discussion}

Although this is a small pilot study, to our knowledge, this is the first multicenter, single-arm, open-label feasibility trial of chemotherapy combined with sintilimab in the treatment of resectable EC. The primary endpoint was achieved, with a pCR of $35.3 \%$. Although the pCR was slightly lower than those in the CORSS study (49\% for squamous cancer) and the 5010 study $(43.2 \%)(8,13)$, it was much higher than that $(12.8 \%)$ achieved by neoadjuvant chemotherapy alone (14), demonstrating that the combinations of chemotherapy with PD-1 inhibitors are effective. Also, the pCR in our current study was higher than that (22\%) achieved after neoadjuvant treatment with chemotherapy plus PD-1 inhibitors in a retrospective study conducted by Fan et al., which might be due to the fact that some patients received only 2 cycles of PD-1 inhibitors preoperatively and their patients had a more advanced disease (15). In a previously reported similar study, patients with ESCC received albumin-bound paclitaxel, carboplatin, plus a PD-1 inhibitor, the pCR was $33.3 \%$, which was slightly lower than in our current study and might again be explained by the limited number of cycles given prior to surgery (16). In our previous study on neoadjuvant treatment in patients with lung cancer, pCR was lower in patients who received 1-2-cycle compared to 
Page 6 of 8

Table 2 Surgical information and postoperative complications

\begin{tabular}{|c|c|}
\hline Parameters & Value \\
\hline Harvested lymph nodes [No.] & $25.3[10-43]$ \\
\hline \multicolumn{2}{|l|}{ Extent of resection } \\
\hline Ro & 16 \\
\hline $\mathrm{R} 1$ & 1 \\
\hline Operative time (min) & 350.9 [185-550] \\
\hline Blood loss (mL) & $165[50-500]$ \\
\hline Postoperative hospital stays (day) & $18.5[8-92]$ \\
\hline \multicolumn{2}{|l|}{ Post-neoadjuvant stage } \\
\hline I & 11 \\
\hline II & 3 \\
\hline IIIA & 1 \\
\hline IIIB & 2 \\
\hline \multicolumn{2}{|l|}{ Postoperative complications } \\
\hline Pneumonia & 6 \\
\hline Hoarseness & 5 \\
\hline Heart failure & 4 \\
\hline Respiratory failure & 2 \\
\hline Anastomotic leakage & 2 \\
\hline Hoarseness & 2 \\
\hline ARDS & 2 \\
\hline
\end{tabular}

The data are shown as $\mathrm{n}$ or $\bar{x} \pm \mathrm{SD}$. ypTNM, extent of tumor presence at time of review. ARDS, acute respiratory distress syndrome. the one receiving 3-4-cycle (7). Although ideas generating, the optimal neoadjuvant treatment duration needs to be confirmed in randomized studies.

Our current study showed the addition of immune checkpoint inhibitor to neoadjuvant therapy is safe as well; there were no deaths within 90 days postoperatively and postoperative complication rates were lower than those of the PALACE-1 trial where grade 3 and higher AEs higher which may be related to the use concurrent chemoradiotherapy. On the other hands, postoperative complications were similar to those of previous neoadjuvant trials in lung cancer. The incidence of grade 3 and higher AEs was similar to the

Table 3 Adverse events of the therapy

\begin{tabular}{lcccc}
\hline Adverse events & Any grade & Grade 1-2 & Grade 3 & Grade 4 \\
\hline Anemia & 3 & 2 & 1 & - \\
Leukopenia & 12 & 8 & 4 & - \\
Neutropenia & 9 & 6 & 3 & - \\
Vomiting & 13 & 11 & 2 & - \\
Diarrhea & 3 & 3 & - & - \\
Fatigue & 8 & 8 & - & - \\
Alopecia & 7 & 6 & 1 & - \\
Arthralgia and bone & 6 & 6 & - & - \\
pain & & & & - \\
Hypothyroidism & 2 & 2 & - & - \\
Rash & 4 & 4 & - & - \\
\hline
\end{tabular}
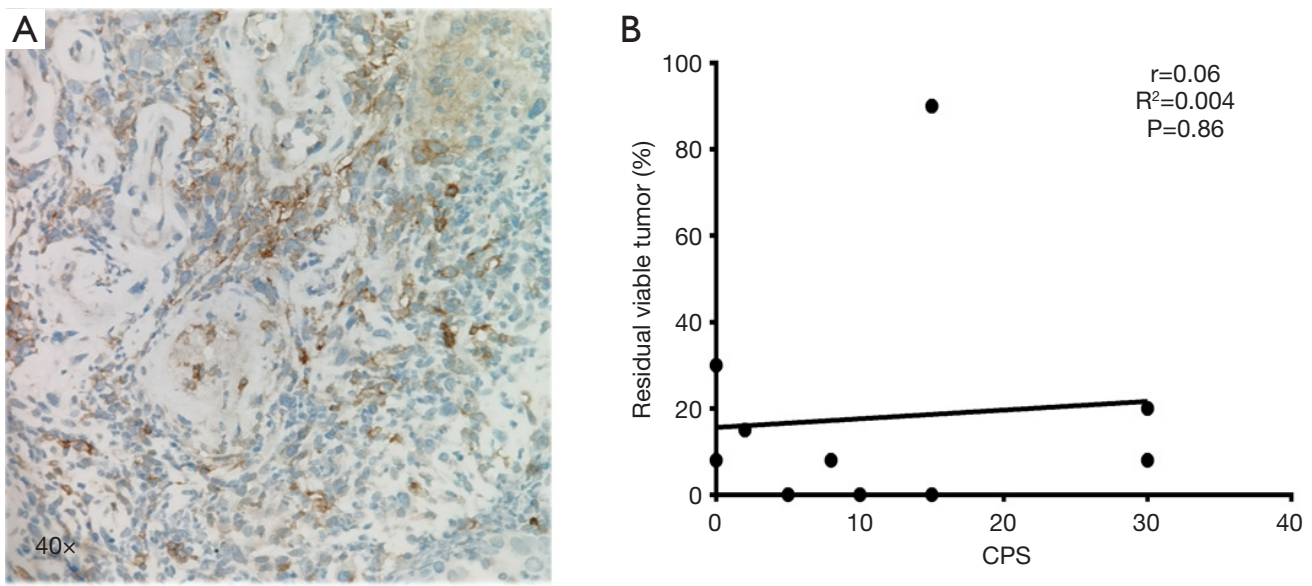

Figure 2 Relationship between PD-L1 expression and RVT. (A) The pre-treated specimen represents the typical IHC image of PD-L1. Magnification 40×; (B) relationship between PD-L1 expression and RVT. CPS, combined positive score; PD-L1, programmed death-ligand 1; RVT, residual viable tumor; IHC, immunohistochemistry. 
NADIM study (30\%), and a phase 2 trial on neoadjuvant atezolizumab and chemotherapy in patients with resectable non-small cell lung cancer $(50 \%)(6,11,17)$. In addition, neoadjuvant chemotherapy alone did not affect the number of LN harvested during surgery. In our current study, the average number of LNs removed was 25.3. Studies have shown that patients will have an OS benefit if they have 21 or more LNs removed after radical surgery for EC $(18,19)$.

Our current study had some limitations: first, although the efficacy and safety of this combination has been preliminarily demonstrated, these findings need to be further confirmed in III stage randomized trial. Secondly, the follow up duration was short and no mature OS data are and third, and the era of predictive markers and based on growing body of literature on PD-L1 expression and response to immune checkpoint inhibitors, evaluating PD-1 expression and its surrogates and stratifying patients based on their CPS score in a large trial is a must.

In conclusion, the addition of sintilimab to preoperative chemotherapy for ESCC is safe and is associated with good response compared to historical data. This combination might spare the patient the toxicity of concurrent radiation. Further testing in large randomized trial is warranted.

\section{Acknowledgments}

The authors appreciate the academic support from the AME Esophageal Cancer Collaborative Group.

Funding: This work was supported by the National Natural Science Foundation of China (82173252, 81871866), and Project of Tangdu Hospital, The Fourth Military Medical University (2018 Key Talents).

\section{Footnote}

Reporting Checklist: The authors have completed the TREND reporting checklist. Available at https://dx.doi. org/10.21037/atm-21-6102

Data Sharing Statement: Available at https://dx.doi. org/10.21037/atm-21-6102

Conflicts of Interest: All authors have completed the ICMJE uniform disclosure form (available at https://dx.doi. org/10.21037/atm-21-6102). The authors have no conflicts of interest to declare.

Ethical Statement: The authors are accountable for all aspects of the work in ensuring that questions related to the accuracy or integrity of any part of the work are appropriately investigated and resolved. All procedures performed in this study involving human participants were in accordance with the Declaration of Helsinki (as revised in 2013). The treatment protocol was approved by the Ethics Committee of Tangdu Hospital of the Air Force Medical University (approval number: 202005-12-KY-07-XW-01), and all participants and their families signed informed consent forms.

Open Access Statement: This is an Open Access article distributed in accordance with the Creative Commons Attribution-NonCommercial-NoDerivs 4.0 International License (CC BY-NC-ND 4.0), which permits the noncommercial replication and distribution of the article with the strict proviso that no changes or edits are made and the original work is properly cited (including links to both the formal publication through the relevant DOI and the license). See: https://creativecommons.org/licenses/by-nc-nd/4.0/.

\section{References}

1. Mao YS, Gao SG, Wang Q, et al. Analysis of a registry database for esophageal cancer from high-volume centers in China. Dis Esophagus 2020;33:doz091.

2. Kojima T, Shah MA, Muro K, et al. Randomized Phase III KEYNOTE-181 Study of Pembrolizumab Versus Chemotherapy in Advanced Esophageal Cancer. J Clin Oncol 2020;38:4138-48.

3. Kato K, Shah MA, Enzinger P, et al. KEYNOTE-590: Phase III study of first-line chemotherapy with or without pembrolizumab for advanced esophageal cancer. Future Oncol 2019;15:1057-66.

4. Jiang H, Zheng Y, Qian J, et al. Efficacy and safety of sintilimab in combination with chemotherapy in previously untreated advanced or metastatic nonsquamous or squamous NSCLC: two cohorts of an open-label, phase $1 \mathrm{~b}$ study. Cancer Immunol Immunother 2021;70:857-68.

5. Gao S, Li N, Gao S, et al. Neoadjuvant PD-1 inhibitor (Sintilimab) in NSCLC. J Thorac Oncol 2020;15:816-26.

6. Provencio M, Nadal E, Insa A, et al. Neoadjuvant chemotherapy and nivolumab in resectable non-small-cell lung cancer (NADIM): an open-label, multicentre, singlearm, phase 2 trial. Lancet Oncol 2020;21:1413-22.

7. Duan H, Wang T, Luo Z, et al. Neoadjuvant programmed cell death protein 1 inhibitors combined with chemotherapy in resectable non-small cell lung cancer: 
an open-label, multicenter, single-arm study. Transl Lung Cancer Res 2021;10:1020-8.

8. Shapiro J, van Lanschot JJB, Hulshof MCCM, et al. Neoadjuvant chemoradiotherapy plus surgery versus surgery alone for oesophageal or junctional cancer (CROSS): long-term results of a randomised controlled trial. Lancet Oncol 2015;16:1090-8.

9. Kumagai K, Rouvelas I, Tsai JA, et al. Meta-analysis of postoperative morbidity and perioperative mortality in patients receiving neoadjuvant chemotherapy or chemoradiotherapy for resectable oesophageal and gastrooesophageal junctional cancers. Br J Surg 2014;101:321-38.

10. Reynolds JV, Preston SR, O'Neill B, et al. ICORG 10-14: NEOadjuvant trial in Adenocarcinoma of the oEsophagus and oesophagoGastric junction International Study (NeoAEGIS). BMC Cancer 2017;17:401.

11. Li C, Zhao S, Zheng Y, et al. Preoperative pembrolizumab combined with chemoradiotherapy for oesophageal squamous cell carcinoma (PALACE-1). Eur J Cancer 2021;144:232-41.

12. Shah MA, Almhanna K, Iqbal S, et al. Multicenter, randomized phase II study of neoadjuvant pembrolizumab plus chemotherapy and chemoradiotherapy in esophageal adenocarcinoma (EAC). J Clin Oncol 2021;39:abstr 4005.

13. Yang H, Liu H, Chen Y, et al. Neoadjuvant Chemoradiotherapy Followed by Surgery Versus Surgery Alone for Locally Advanced Squamous Cell Carcinoma of the Esophagus (NEOCRTEC5010): A Phase III Multicenter, Randomized, Open-Label Clinical Trial. J

Cite this article as: Duan H, Wang T, Luo Z, Wang X, Liu H, Tong L, Dong X, Zhang Y, Valmasoni M, Kidane B, Almhanna K, Wiesel O, Pang S, Ma J, Yan X. A multicenter single-arm trial of sintilimab in combination with chemotherapy for neoadjuvant treatment of resectable esophageal cancer (SIN-ICE study). Ann Transl Med 2021;9(22):1700. doi: 10.21037/atm-21-6102
Clin Oncol 2018;36:2796-803.

14. Ancona E, Ruol A, Santi S, et al. Only pathologic complete response to neoadjuvant chemotherapy improves significantly the long term survival of patients with resectable esophageal squamous cell carcinoma: final report of a randomized, controlled trial of preoperative chemotherapy versus surgery alone. Cancer 2001;91:2165-74.

15. Fan M, Dai L, Yan W, et al. Efficacy of programmed cell death protein 1 inhibitor in resection transformation treatment of esophageal cancer. Thorac Cancer 2021;12:2182-8.

16. Shen D, Chen Q, Wu J, et al. The safety and efficacy of neoadjuvant PD-1 inhibitor with chemotherapy for locally advanced esophageal squamous cell carcinoma. J Gastrointest Oncol 2021;12:1-10.

17. Shu CA, Gainor JF, Awad MM, et al. Neoadjuvant atezolizumab and chemotherapy in patients with resectable non-small-cell lung cancer: an open-label, multicentre, single-arm, phase 2 trial. Lancet Oncol 2020;21:786-95.

18. van der Schaaf M, Johar A, Wijnhoven B, et al. Extent of lymph node removal during esophageal cancer surgery and survival. J Natl Cancer Inst 2015;107:djv043.

19. Hagens ERC, van Berge Henegouwen MI, Cuesta MA, et al. The extent of lymphadenectomy in esophageal resection for cancer should be standardized. J Thorac Dis 2017;9:S713-23.

(English Language Editor: J. Jones) 